\title{
L-BAND DIGITAL AERONAUTICAL COMMUNICATIONS SYSTEM (LDACS) FLIGHT TRIALS IN THE NATIONAL GERMAN PROJECT MICONAV
}

\author{
Thomas Gräupl, Nicolas Schneckenburger, Thomas Jost, Michael Schnell, Alexandra Filip, Miguel A. \\ Bellido-Manganell, Daniel M. Mielke, Nils Mäurer, Rachit Kumar, Okuary Osechas, Giuseppe \\ Battista, German Aerospace Center (DLR), Institute of Communications and Navigation, \\ Oberpfaffenhofen-Wessling, Germany \\ Thomas Bögl, Thomas Richter, Rohde \& Schwarz, München, Germany
}

\begin{abstract}
Today's voice-based air-ground communication system for aircraft guidance is suffering from increasing saturation of the VHF band in high density areas. Therefore the European Union strives for a transition from analog voice communication to more spectrum efficient digital communication. This transition shall be realized, among others, through the development and implementation of the L-band Digital Aeronautical Communications System (LDACS). In order to verify the suitability of LDACS for both communications and navigation, a flight trial campaign will be performed within the nationally funded German project MICONAV. The objective of this paper is to present the planned LDACS measurement campaign. LDACS will be validated under realistic conditions: Four ground stations transmit signals; one airborne station onboard an aircraft will receive the communication messages and additionally utilize the signals from the ground stations for navigation.
\end{abstract}

\section{Introduction}

Today's VHF voice-based air-ground communication system for aircraft guidance is suffering from increasing saturation of the VHF band in high density areas [1]. Therefore, the European Union (EU) strives for a sustainable modernization of the aeronautical communication infrastructure.

In the long-term, air traffic management communication is expected to transition from analog VHF voice communication to a more spectrum efficient digital data communication alternative. The European ATM Master Plan [2] foresees this transition to be realized, among others, through the development and implementation of the L-band Digital Aeronautical Communications System (LDACS).
The development of LDACS has already made substantial progress: An LDACS system specification has been produced [3]; transmitter demonstrators were developed to prove the spectrum compatibility of LDACS with existing systems operating in the Lband [4]; and the overall system performance has been analyzed with the help of computer simulations, indicating that LDACS can fulfil the identified requirements [5]. In addition, the LDACS standardization process within ICAO has been initiated in December 2016 and is currently in progress.

However, LDACS has not yet been demonstrated outside of the laboratory. In order to verify the suitability of the LDACS system for both communications and navigation, a flight trial campaign will be performed within the nationally funded German project MICONAV (Migration towards Integrated COM/NAV Avionics).

The objective of this paper is to present the planned LDACS measurement campaign. In preparation of this measurement campaign, prototypes of the LDACS ground stations and airborne stations have been implemented by the MICONAV consortium. These prototypes will be used to validate the LDACS performance under realistic conditions: Four ground stations transmit LDACS signals; one airborne station onboard an aircraft will receive the communication messages and additionally utilize the signals from the ground stations for navigation.

\section{Background on LDACS}

LDACS is a broadband air-ground datalink proposed to supplement the VHF communication infrastructure in the L-band [6]. It is designed to provide air-ground data communication with optional support for digital voice. It is a cellular broadband 
system based on Orthogonal Frequency-Division Multiplexing (OFDM) technology [7] and supports quality-of-service while taking the requirements of aeronautical services into account. Moreover, it shares many technical features with $3 \mathrm{G}$ and $4 \mathrm{G}$ wireless communications systems. In addition to communication, LDACS supports navigation with its built-in ranging functionality.

LDACS will be one of several wireless access networks connecting aircraft to the aeronautical telecommunications network. The LDACS access network contains several ground stations, each of them providing one LDACS radio cell for communication and ranging.

The LDACS air interface is a cellular datalink with a star-topology connecting aircraft to ground stations with a full duplex radio link. Each ground station is the centralized instance controlling all airground communications within its radio cell.

The LDACS protocol stack defines two layers, the physical layer and the data link layer.

The physical layer provides the means to transfer data over the radio channel. The LDACS ground station supports bi-directional links to multiple aircraft under its control. The forward link direction (FL; ground-to-air) and the reverse link direction (RL; air-to-ground) are separated by frequency division duplex. Forward link and reverse link use a $500 \mathrm{kHz}$ channel each. The ground station transmits a continuous stream of OFDM symbols on the forward link. In the reverse link different aircraft are separated in time and frequency using a combination of orthogonal frequency-division multiple-access and time-division multiple-access. Aircraft thus transmit discontinuously on the reverse link with radio bursts sent in precisely defined transmission opportunities allocated by the ground station.

The data-link layer provides the necessary protocols to facilitate concurrent and reliable data transfer for multiple users. The LDACS data link layer is organized in two sub-layers: The medium access sub-layer and the logical link control sublayer. The medium access sub-layer manages the organization of transmission opportunities in slots of time and frequency. The logical link control sublayer provides reliable and acknowledged point-topoint logical channels between the aircraft and the ground station using an automatic repeat request protocol.

\section{Background on MICONAV}

MICONAV is a research project receiving national funding from the research program LuFo V2 (Luftfahrt-Forschungsprogramm) of the German Federal Ministry of Economy and Energy (BMWi).

The goals of the project MICONAV (Migration towards Integrated COM/NAV Avionics) are twofold: First, a fully functional LDACS demonstrator as defined within the LDACS specification [3] is developed and realized using industrial development methods. Second, LDACS ranging functionality is developed and implemented to support alternative positioning navigation and timing (APNT) [8]. Utilizing LDACS for navigation in addition to communication offers favorable synergy effects and minimizes costs: having deployed LDACS as a communications system, no additional infrastructure will be needed for APNT. The objectives of the project are summarized in Figure 1

MICONAV builds on the results of two previous projects: ICONAV $^{1}$ and LDACS-NAV ${ }^{2}$. It is conducted in liaison with SESAR2020. The results of the project are therefore communicated to interested organizations such as EUROCONTROL and ICAO.

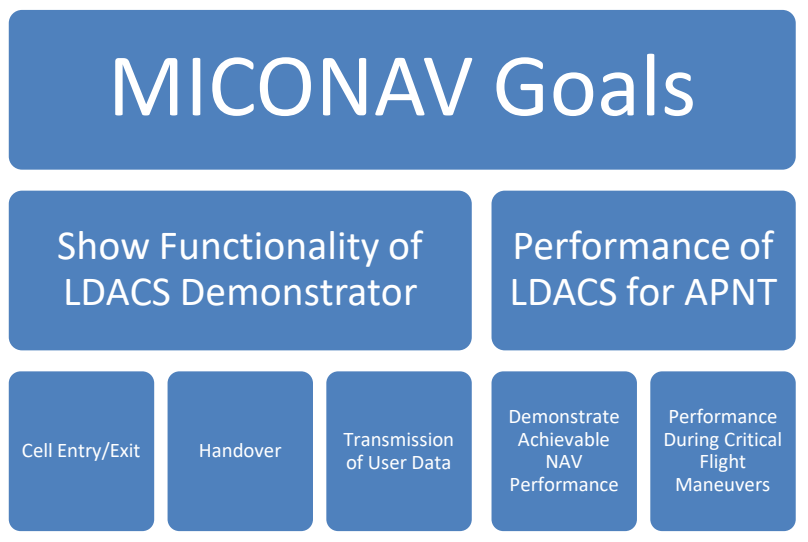

Figure 1. Objectives of the MICONAV Project

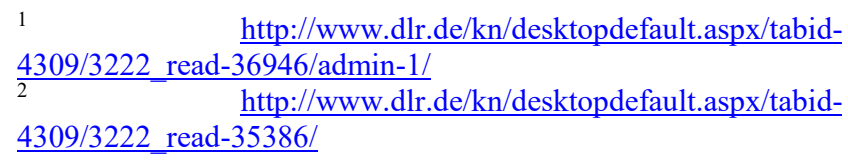




\section{Method}

In the project MICONAV in-flight measurements of LDACS will be conducted in late 2018 demonstrating LDACS communication and navigation in a realistic scenario.

The flight trial campaign will feature one airborne LDACS system installed in a research aircraft. The airborne station will have four counterparts on the ground: Two LDACS ground stations providing full communication and navigation functionality, and two additional ground stations providing only navigation functionality.

\section{Validation Objectives}

The objective of the flight trials is to verify the functionality of LDACS, measure the performance of LDACS, and demonstrate the benefits of LDACS. The flight trials will also be supported by preparatory lab trials.

\section{Communication}

The communication function of LDACS will be validated qualitatively according to its functionality, and quantitatively according to its performance.

The qualitative validation of LDACS focuses on the correct functionality of its main control plane and user plane communication functions. In particular the following features will be validated: Cell entry, cell exit, handover between ground stations, and the transmission of user data.

The quantitative validation of the LDACS communication functionality focuses on the control plane and user plane performance. The key performance indicators for the control plane performance are the duration of cell entry and handover. In the user plane the latency and throughput of user data transmissions of varying classes of service will be measured.

Communication data generating load for LDACS are simple services like file transfers and custom scripts generating traffic patterns suitable for measurement. Logging information is provided by the system via UDP.

\section{Navigation}

LDACS is also envisioned as an alternative navigation system with expected performance to support navigation performance of RNP 0.3 and higher. The navigation performance will be characterized by the positioning error, which depends on the ranging performance as well as on the geometry of the ground stations as seen from the aircraft. Of particular interest is the navigation performance in challenging flight situations, e.g., when banking or flying at low altitudes.

For the MICONAV flight trails, the achievable navigation performance of LDACS will be demonstrated by using four ground stations transmitting LDACS signals. Given the current conception of LDACS as a pseudo-ranging system, the ground infrastructure for the flight trials will need to be well synchronized. In the next section we provide a short overview of the time synchronization method, followed by a description of the software used to assess the navigation performance in real time.

\section{Ground Station Synchronization}

The pseudo-ranging nature of LDACS requires tight synchronization between the ground stations. For the flight trials, this will be achieved by synchronizing the ground stations to GPS time. According to [16] GPS-based time synchronization is able to achieve $50 \mathrm{~ns}$ or better in real time. The accuracy can be improved further in post-processing.

In the experimental implementation the LDACS signals do not transmit navigation data. Hence a predefined template signal will be used for correlation processing, which is part of a super-frame (SF). The SF of an LDACS signal is $240 \mathrm{~ms}$ long, a multiple of a second is achieved after 25 SFs. These $25 \mathrm{SF}$ correspond to a time of 6 seconds. To align the frame structure of all ground stations, the ground stations have to be started either exactly at the same time or at a multiple of 6 seconds to each other. Therefore, it is necessary to generate trigger signal in the cycle of six seconds (pulse per 6 seconds - PP6S). Separate circuitry is implemented to achieve simultaneous PP6S at all four stations. The logic block diagram of the circuitry is shown in Figure 2.

The trigger control unit (TCU) is implemented using a microcontroller. It receives the GPS time information from a GPS receiver using a Serial Peripheral Interface (SPI). The TCU generates a trigger control signal (TCS) at the modulo-six of GPS time. This operation ensures simultaneous PP6S generation at all stations. The trigger is used to open the AND gate, allowing the PPS from the atomic 
clock to pass through. The PP6S generation concept is illustrated in Figure 3.

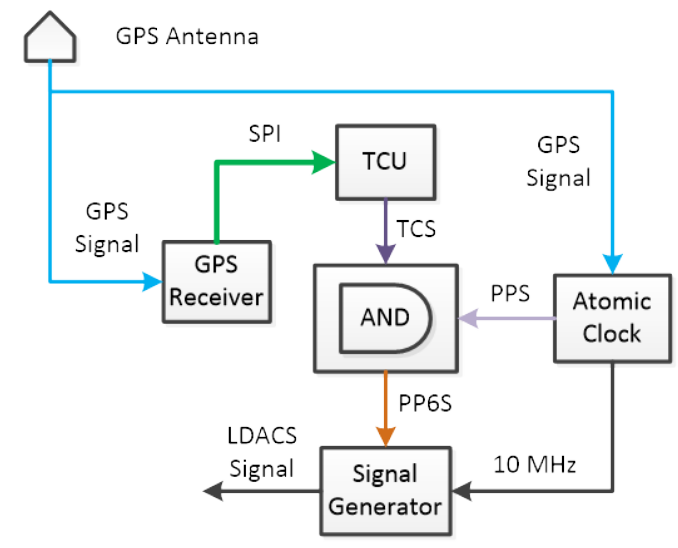

Figure 2. Block Diagram of PP6S Logic

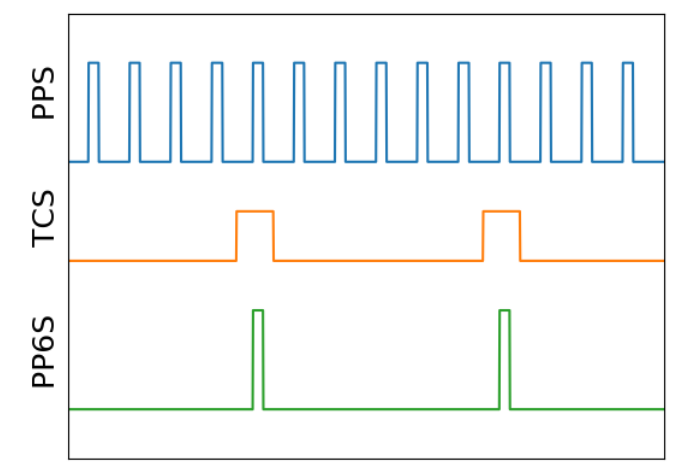

Figure 33 Masking of PPS by TCS to Generate PP6S

\section{Real-Time Positioning}

In the MICONAV flight trail we aim to perform real-time positioning using LDACS signals. The positioning performance will be assessed in software. The software receives the navigation data over UDP from the signal processing unit. Received data is processed to obtain a fix which can be displayed in real time. The software also displays positioning errors using GPS as ground truth.

\section{Post-Processing}

Since LDACS is not a fully developed system for navigation, post-processing of data may be used to remove the effect of clock-synchronization error to assess the actual performance of the system. In postprocessing the time synchronization error of the ground stations is minimized, which results in position estimates more realistic for the final system. Further, we may also use a tropospheric error model and Doppler smoothing to improve the positioning performance of the LDACS system to verify the limits of the future deployed system.

\section{System under Test}

To simplify development, the LDACS communication and navigation receivers and transmitters are split into separate devices. All devices are realized on the WFDE Rohde\&Schwarz development platform. The platform consists of an RF (WFDE-TU) and digital (WFDE-DU) unit. Both RF and digital units are shown in Figure 4.

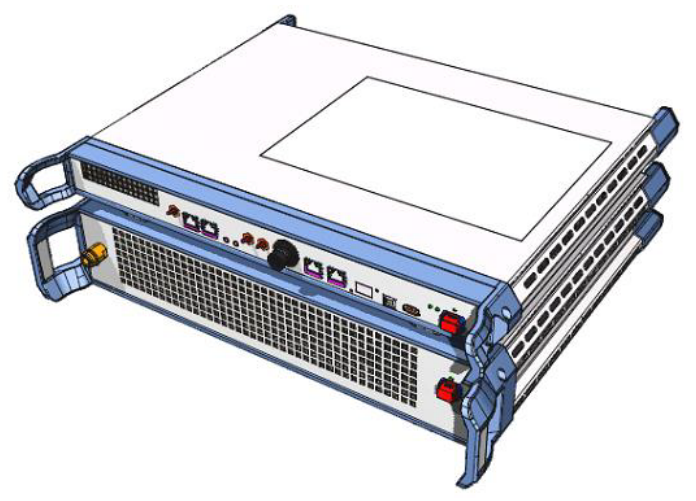

Figure 4: Rohde\&Schwarz Development Environment - Digital (Top) and RF (Bottom) Unit

The principal hardware setup within the aircraft is depicted in Figure 5. All digital units are controlled by a computer. The computer features a basic application allowing simple services like file transfers using LDACS. Additionally, the aircraft position is calculated in real time on the computer. The GPS receiver onboard the aircraft acts both as position as well as time reference for later evaluation.

The ground stations' setup is very similar to the aircraft setup. The main difference is that the ground stations do not feature a navigation receiver.

To allow for the airborne navigation receiver to determine its position based on the LDACS signal, all ground stations have to be time synchronized. The synchronization is realized using GPS disciplined Rubidium atomic clocks. The chosen method is expected to provide a synchronization accuracy in the order of 10-50 ns in real time (see section "Ground Station Synchronization). 


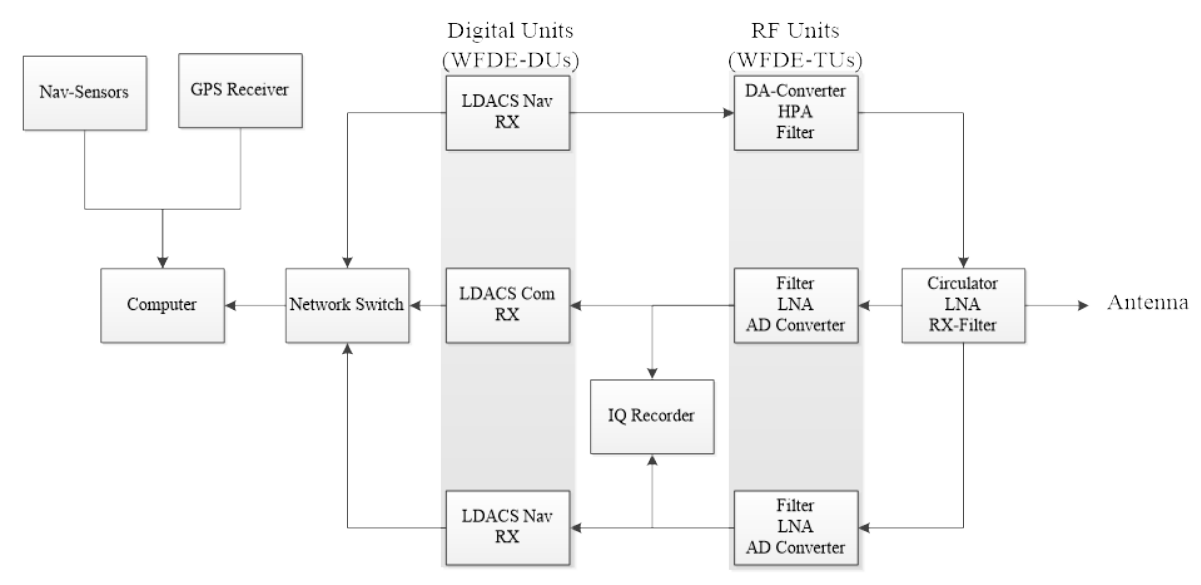

Figure 5. Hardware Setup Inside the Aircraft

\section{Test Scenarios}

Project MICONAV focuses on two types of test scenarios: Laboratory trials for preparation, and flight trials. Preparatory laboratory trials are performed to ascertain that the system has been integrated correctly and that all the required functions work as expected. Flight trials will be carried out on board the aircraft (on-ground or flying) after the lab trials to present the benefits of LDACS in a realistic environment.

During the laboratory tests the basic functionality of the LDACS prototype is evaluated. These tests include an analysis of the spectrum and of the SNR dependence of both the communication and navigation performance. Additionally, the synchronization error between the different ground stations is analyzed.

For the flight trials, three different types of flight routes have been planned. In the following we illustrate each type by an example.

Figure 6 shows the first planned flight route type, where the continuous blue line represents the main route, which has to be repeated for different flight altitudes. The red dots represent the ground stations. The purpose of this flight pattern is to test the navigation capabilities of LDACS for a rich variety of geometries and distances to the different ground stations. Moreover, the flight route shown in Figure 6 allows us to test advanced communication functionalities of LDACS like handovers between ground stations in a realistic scenario.

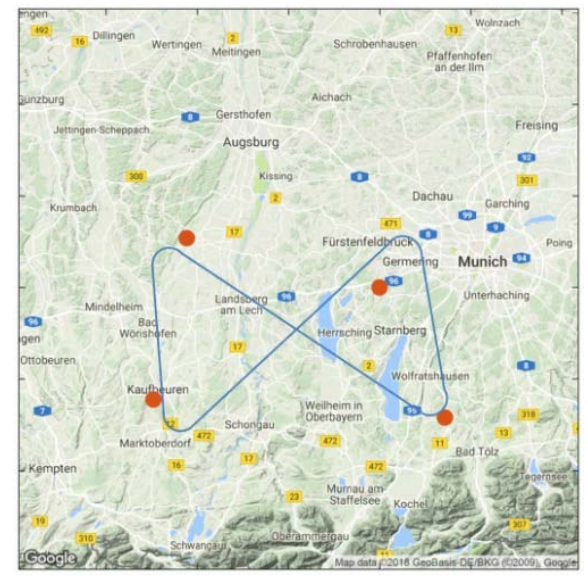

\section{Figure 6. Butterfly Route Scenario, Map Dimensions 120 x $120 \mathrm{~km}$ (c) Google}

Figure 7 depicts the second planned flight route type. After taking off, the aircraft will fly in a circle to emulate a banking turn. Data obtained during this flight route will be useful to test communication and navigation capabilities during the takeoff and landing phase.

Figure 8 shows the third planned flight route type. In this case, the route is followed until the received signal strength is so low that messages transmitted from the ground stations cannot be decoded anymore. At that point, the plane will return to the airport. Note that Figure 8 only represents an example of the flight route type and that the real point of return may differ. 


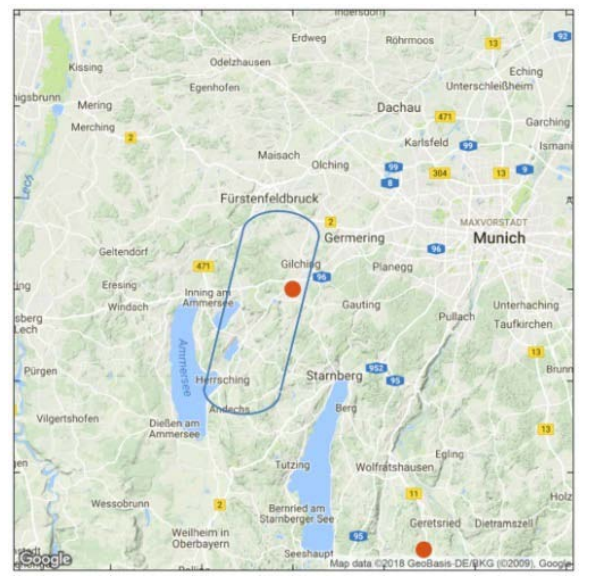

Figure 7. Banking Route Scenario, Map Dimensions 60 x 60 km @ Google

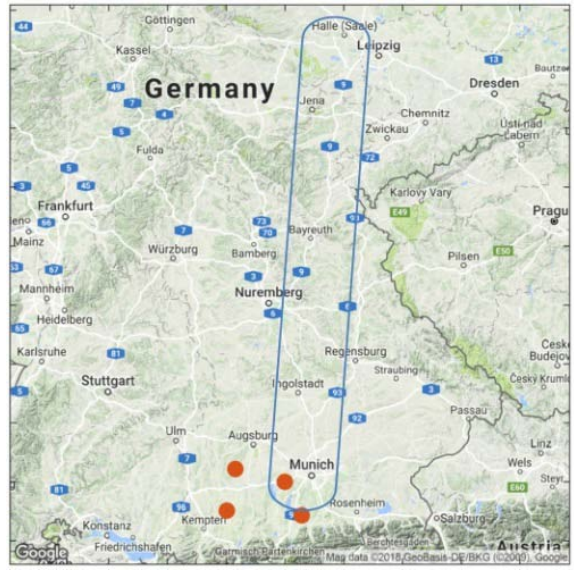

Figure 8. Long Route Scenario, Map Dimensions $475 \times 475$ km (C) Google

\section{Conclusion}

In order to verify the suitability of LDACS for both communications and navigation, a flight trial campaign will be performed within the nationally funded German project MICONAV.

The objective of this paper was to present this planned measurement campaign. LDACS will be validated under realistic conditions: Four ground stations transmit signals; one airborne station onboard an aircraft will receive the communication messages and additionally utilize the signals from the ground stations for navigation.

The objective of the flight trials is to verify the functionality of LDACS, measure its performance, and demonstrate its benefits. The flight trials will also be supported by preparatory lab trials.

\section{References}

[1] B. Kamali, "An Overview of VHF Civil Radio Network and the Resolution of Spectrum Depletion," in 2010 Integrated Communications, Navigation, and Surveillance Conference Proceedings, ICNS 2010, 2010.

[2] SESAR JU, "European ATM Master Plan," 2012. [Online]. Available: https://www.atmmasterplan.eu/download/29. [Accessed: 26-Jan-2015].

[3] M. Sajatovic, B. Haindl, U. Epple, T. Gräupl, C. Rihacek, M. Schnell, N. Fistas, J.-U. Koch, H.-W. Kim, and E. Le-Ho, "EWA04-1-T2-D1 Updated LDACS1 System Specification," Brussels, Belgium, 2011.

[4] M. Sajatovic, H. Günzel, and S. Müller, "WA04 D22 Test Report for Assessing LDACS1 Transmitter Impact upon DME/TACAN Receivers," Brussels, Belgium, 2014.

[5] F. Hoffmann, U. Epple, M. Schnell, and U. Fiebig, "Feasibility of LDACS1 Cell Planning in European Airspace," in Digital Avionics Systems Conference (DASC), 2012 IEEE/AIAA 31st, 2012, p. 5E1-1-5E1-13.

[6] M. Schnell, U. Epple, D. Shutin, and N. Schneckenburger, "LDACS: Future Aeronautical Communications for Air-Traffic Management," Commun. Mag. IEEE, vol. 52, no. 5, pp. 104-110, 2014.

[7] S. Brandes, U. Epple, S. Gligorevic, M. Schnell, B. Haindl, and M. Sajatovic, "Physical Layer Specification of the L-Band Digital Aeronautical Communications System (L-DACS1)," in Proceedings of the 2009 Integrated Communications, Navigation and Surveillance Conference, ICNS 2009, 2009.

[8] L. Eldredge, P. Enge, M. Harrison, R. Kenagy, S. Lo, R. Loh, R. Lilley, M. Narins, and R. Niles, "Alternative Positioning, Navigation and Timing (PNT) Study," in International Civil Aviation Organisation Navigation Systems Panel (NSP), 2010. 


\section{Acknowledgements}

This work was co-funded under the research program LuFo - V (Luftfahrt-Forschungsprogramm) of the German Federal Ministry of Economy and Energy BMWi.

\section{Acronyms and Abbreviations}

$\mathrm{A} / \mathrm{C} \quad$ Aircraft

A/G Air/Ground

APNT Alternative Navigation Positioning and Timing

ATM Air Traffic Management

EU European Union

GPS Global Positioning System

GS Ground station
LDACS L-band Digital Aeronautical Communication System

PP6S Pulse Per 6 Seconds

RF Radio Frequency

RNP Required Navigation Performance

SF $\quad$ Super Frame

SPI Serial Peripheral Interface

TCS Trigger Control Signal

TCU Trigger Control Unit

UDP User Datagram Protocol

WFDE Waveform Development Environment

2018 Integrated Communications Navigation and Surveillance (ICNS) Conference

April 10-12, 2018 